\title{
Critical point equation on almost $f$-cosymplectic manifolds
}

\author{
H. Aruna Kumara and V. Venkatesha \\ Department of Mathematics, Kuvempu University, Shimoga, India, and \\ Devaraja Mallesha Naik \\ Department of Mathematics, CHRIST (Deemed to be University), Bengaluru, India
}

\begin{abstract}
Purpose - Besse first conjectured that the solution of the critical point equation (CPE) must be Einstein. The CPE conjecture on some other types of Riemannian manifolds, for instance, odd-dimensional Riemannian manifolds has considered by many geometers. Hence, it deserves special attention to consider the CPE on a certain class of almost contact metric manifolds. In this direction, the authors considered $\mathrm{CPE}$ on almost f-cosymplectic manifolds.

Design/methodology/approach - The paper opted the tensor calculus on manifolds to find the solution of the CPE.

Findings - In this paper, in particular, the authors obtained that a connected $f$-cosymplectic manifold satisfying CPE with \lambda= $=$ tilde $\{f\}$ is Einstein. Next, the authors find that a three dimensional almost $f$-cosymplectic manifold satisfying the $\mathrm{CPE}$ is either Einstein or its scalar curvature vanishes identically if its Ricci tensor is pseudo anti-commuting.

Originality/value - The paper proved that the $\mathrm{CPE}$ conjecture is true for almost $f$-cosymplectic manifolds. Keywords Critical point equation, Almost $f$-cosymplectic manifold, Cosymplectic manifold, Einstein manifold Paper type Research paper
\end{abstract}

\section{Introduction}

One of the natural ways of finding canonical Riemannian metric, that is, Riemannian metrics with constant curvature in various form on a smooth manifold is to look for metrics which are critical points of a natural functional on the space of all metrics on a given manifold. In this context, it is very interesting to investigate the critical points of total scalar curvature functional $\mathcal{S}: \mathcal{M} \rightarrow \mathbb{R}$ given by

$$
\mathcal{S}(g)=\int_{M} r_{g} d v_{g}
$$

defined on a compact orientable Riemannian $n$-manifold $(M, g)$, where $\mathcal{M}$ denotes set of all Riemannian metrics on $(M, g)$ of unit volume, $r_{g}$ is the scalar curvature and $d v_{g}$ is the volume form. The functional $\mathcal{S}$ in Eqn (1.1) restricted over $\mathcal{M}$ is known as Einstein-Hilbert functional and its critical points are the Einstein metric (see chapter 2 in [1]).

\section{JEL Classification - 53C15, 53C20, 553C21}

(C) H. Aruna Kumara, V. Venkatesha and Devaraja Mallesha Naik. Published in Arab Journal of Mathematical Sciences. Published by Emerald Publishing Limited. This article is published under the Creative Commons Attribution (CC BY 4.0) licence. Anyone may reproduce, distribute, translate and create derivative works of this article (for both commercial and non-commercial purposes), subject to full attribution to the original publication and authors. The full terms of this licence may be seen at http:// creativecommons.org/licences/by/4.0/legalcode

H.A. Kumara and V. Venkatesha are thankful to Department of Science and Technology, New Delhi for financial assistance to the Department of Mathematics, Kuvempu University under theFISt program (Ref. No. SR/FST/MS-I/2018-23(C)).
Received 21 October 2020 Revised 4 February 2021 Accepted 4 February 2021 
Let $\mathcal{C} \subset \mathcal{M}$ be the subset of metrics with constant scalar curvature. If we consider the functional in Eqn (1.1) restricted to $\mathcal{C}$, then it is not difficult to see that the Euler-Lagrangian equation is given by,

$$
\operatorname{Hess}_{g} \lambda-\left(\Delta_{g} \lambda\right)-\lambda \operatorname{Ric}_{g}=\operatorname{Ric}_{g}-\frac{r}{n} g
$$

for some smooth function $\lambda$ on $M$. Here Hess, $\Delta_{g}$, Ric and $r$ stands for the Hessian form, the Laplacian, the Ricci tensor and the scalar curvature on $M$, respectively. Moreover, taking trace in Eqn (1.2), we obtain

$$
\Delta_{g} \lambda+\frac{r \lambda}{n-1}=0
$$

We notice that if $\lambda$ is constant in Eqn (1.2), then $\lambda=0$ and $g$ becomes Einstein. Therefore, we have the following definition:

Definition 1.1. A compact Riemannian manifold $(M, g)$ of dimension $n>3$ with constant scalar curvature and unit volume together with a smooth potential function $\lambda$ satisfying (Eqn 1.2), is called critical point equation (shortly, CPE).

Besse first conjectured that the solution of the CPE must be Einstein [1]. Since then, we find many articles regarding the solution of the CPE. In [2], Barros and Ribeiro proved that the $\mathrm{CPE}$ conjecture is true under the assumption of half conformally flat spaces. Recently, Hwang [3] proved that the CPE conjecture is also true under certain condition on the bounds of the potential function $\lambda$. A necessary and sufficient condition for the norm of the gradient of the potential function for a CPE metric to be the Einstein metric was obtained by Neto [4].

It is very interesting to consider the $\mathrm{CPE}$ on odd-dimensional Riemannian manifolds. In this direction, Ghosh and Patra considered the $K$-contact metrics that satisfy the CPE [5], and proved that the CPE conjecture is true for this class of metric. Patra et al. in [6], and De and Mandal in [7] independently considered an almost Kenmotsu manifold with CPE. Recently, present authors in [8], and Blaga and Dey in [9] studied CPE on cosymplectic manifold and three dimensional $\alpha$-cosymplectic manifold, respectively.

As the generalization of almost Kenmotsu and almost cosymplectic manifolds, the results obtained in [6-9] motivates us to consider almost $f$-cosymplectic manifolds. In this paper, we classify an almost $f$-cosymplectic manifold which satisfies $\mathrm{CPE}$.

\section{Preliminaries}

Let $M$ be a smooth differentiable manifold of dimension $2 n+1$ equipped with a triple $(\varphi, \xi, \eta)$, where $\varphi$ is a (1,1)-tensor field, $\xi$ is a Reeb vector and $\eta$ is a one-form such that

$$
\varphi^{2} X=-X+\eta(X) \xi, \quad \eta(\xi)=1,
$$

which implies $\varphi(\xi)=0, \eta(\varphi)=0$ and $\operatorname{rank}(\varphi)=2 n$. If $M$ admits a Riemannian metric $g$ such that

$$
g(\varphi X, \varphi Y)=g(X, Y)-\eta(X) \eta(Y), \quad g(X, \xi)=\eta(X),
$$

for any vector fields $X, Y$, then $M$ is said to have an almost contact metric structure $(\varphi, \xi, \eta, g)$. On such a manifold, the fundamental two-form $\Phi$ of $M$ is defined by

$$
\Phi(X, Y)=g(\varphi X, Y),
$$

for any vector field $X$ and $Y$ on $M$. One can define an almost complex structure $J$ on $M \times \mathbb{R}$ by 


$$
J\left(X, u \frac{d}{d t}\right)=\left(\varphi X-u \xi, \eta(X) \frac{d}{d t}\right),
$$

CPE on almost $f$-cosymplectic manifolds

where $t$ is the coordinate of $\mathbb{R}$ and $u$ is a smooth function. If the aforesaid structure $J$ is integrable, then we call an almost contact structure as normal, and this is equivalent to require

$$
[\varphi, \varphi]=-2 d \eta \otimes \xi
$$

where $[\varphi, \varphi]$ indicates the Nijenhuis tensor of $\varphi$.

An almost contact metric manifold $M$ is said to be almost cosymplectic if $d \eta=0$ and $d \Phi=0$, where $d$ is the exterior differential operator, and it is said to be cosymplectic if in addition the almost contact structure is normal. An almost $\alpha$-Kenmotsu manifold is an almost contact metric manifold, in which $d \eta=0$ and $d \Phi=2 \alpha \eta \wedge \Phi$, for a nonzero constant $\alpha$. More generally, if the constant $\alpha$ is any real number, then almost contact structure is said to be almost $\alpha$-cosymplectic [10]. Moreover, the authors in [11] generalizes the almost $\alpha$-cosymplectic manifold by allowing the real number $\alpha$ to any smooth function $f$, and it is called as an almost $f$-cosymplectic manifold, which is an almost contact metric manifold $M$ such that $d \Phi=2 f \eta \wedge \Phi$ and $d \eta=0$ for a smooth function $f$ satisfying $d f \wedge \eta=0$. In addition, a normal almost $f$-cosymplectic manifold is said to be $f$-cosymplectic manifold. In particular, $M$ is an almost cosymplectic manifold under the condition $f$ (constant) $=0$ and an almost $\alpha$-Kenmotsu manifold if $(\alpha=f \neq 1)$.

Besides, we recall that there is an operator $h=\frac{1}{2} £_{\xi} \varphi$, which is a self-dual operator. We denote by $R$ and Ric the Riemannian curvature tensor and Ricci tensor, respectively. For an almost $f$-cosymplectic manifold $M$, the following equations were proved [11]:

$$
\begin{gathered}
\nabla_{X} \xi=-f \varphi^{2} X-\varphi h X, \quad \operatorname{trace}(\varphi h)=0, \\
R(X, \xi) \xi-\varphi R(\varphi X, \xi) \xi=2\left(\tilde{f} \varphi^{2} X-h^{2} X\right), \\
\operatorname{Ric}(\xi, \xi)=-2 n \tilde{f}-\operatorname{trace}\left(h^{2}\right), \\
R(X, \xi) \xi=\tilde{f} \varphi^{2} X+2 f \varphi h X-h^{2} X+\varphi\left(\nabla_{\xi} h\right) X,
\end{gathered}
$$

for any vector fields $X, Y$ on $M$, where $\tilde{f}=\xi(f)+f^{2}$.

\section{CPE on normal almost $f$-cosymplectic manifolds}

In this section, we aim to study CPE on normal almost $f$-cosymplectic manifold. We are aware that if almost contact metric manifold is normal then $h=0$. Hence, as a result of Proposition 9 and Proposition 10 of [11] we have the following identities, which are valid on $f$-cosymplectic manifolds;

$$
\begin{gathered}
\nabla_{X} \xi=-f \varphi^{2} X \\
Q \xi=-2 n \tilde{f} \xi
\end{gathered}
$$




$$
R(X, Y) \xi=\tilde{f}\{\eta(X) Y-\eta(Y) X\},
$$

where $Q$ is the Ricci operator of $M$.

Now, we will give some properties, which will be used in the proof of our results.

Lemma 3.1. An $f$-cosymplectic manifold $M$ of dimension $2 n+1$ satisfies

$$
\begin{gathered}
\left(\nabla_{X} Q\right) \xi=-f Q X-2 n(X \tilde{f}) \xi-2 n \tilde{f} f X, \\
\left(\nabla_{\xi} Q\right) X=-2 f Q X-(2 n-1)(X \tilde{f}) \xi-(\tilde{\xi}) X-4 n \tilde{f} f X .
\end{gathered}
$$

Proof. Differentiation of Eqn (3.2), and utilization of first term of Eqn (3.1) provides Eqn (3.4). Now differentiating Eqn (3.3) along $Z$ leads to

$$
\begin{aligned}
\left(\nabla_{Z} R\right)(X, Y) \xi= & (Z \tilde{f})\{\eta(X) Y-\eta(Y) X\}+\tilde{f} f\{g(X, Z) Y \\
& -g(Y, Z) X\}-f R(X, Y) Z .
\end{aligned}
$$

Taking $X=Z=E_{i}$ in the above equation and then summing over $i$ shows that

$$
\begin{aligned}
\sum_{i=1}^{2 n+1} g\left(\left(\nabla_{E_{i}} R\right)\left(E_{i}, Y\right) \xi, Z\right)= & (\tilde{\xi}) g(Y, Z)-(Z \tilde{f}) \eta(Y) \\
& +2 n \tilde{f} f g(Y, Z)+f \operatorname{Ric}(Y, Z) .
\end{aligned}
$$

One can easily deduce from second Bianchi identity that

$$
\left.\sum_{i=1}^{2 n+1} g\left(\left(\nabla_{E_{i}} R\right)(Z, \xi) Y, E_{i}\right)=g\left(\left(\nabla_{Z} Q\right) \xi, Y\right)-g\left(\nabla_{\xi} Q\right) Z, Y\right) .
$$

Feeding Eqn (3.7) into Eqn (3.6) and with the help of Eqn (3.4), we obtain

$$
\begin{aligned}
g\left(\left(\nabla_{\xi} Q\right) Z, Y\right)= & -2 f \operatorname{Ric}(Z, Y)-(2 n-1)(Z \tilde{f}) \eta(Y) \\
& -(\tilde{\xi}) g(Z, Y)-4 n \tilde{f} f g(Z, Y),
\end{aligned}
$$

which proves Eqn (3.5).

Lemma 3.2. [5] Let $(g, \lambda)$ be a nontrivial solution of the CPE (Eqn 1.2) on $n$-dimensional Riemannian manifold $M$. Then the curvature tensor $R$ can be expressed as

$$
\begin{gathered}
R(X, Y) D \lambda=(X \lambda) Q Y-(Y \lambda) Q X+(\nu+1)\left(\left(\nabla_{X} Q\right) Y-\left(\nabla_{Y} Q\right) X\right) \\
+(X \nu) Y-(Y \nu) X,
\end{gathered}
$$

for any vector fields $X, Y$ on $M$, where $\nu=-r\left(\frac{\lambda}{n-1}+\frac{1}{n}\right)$.

In the following, we will consider an $f$-cosymplectic manifold $M$ satisfying a $C P E$ and assume that the function $f$ satisfies $\xi(\tilde{f})=0$.

Theorem 3.3. Let $M$ be an $f$-cosymplectic manifold of dimension $2 n+1$ with $\xi(\tilde{f})=0$. If $(g, \lambda)$ is a solution of the CPE (Eqn 1.2), then one of the following statement holds:

(1) $M$ is Einstein 
(2) $M$ is locally the product of a Kähler manifold and an interval or unit circle $\mathbb{S}^{1}$.

CPE on almost

Proof. Taking scalar product of Eqn (3.8) with $\xi$ and making use of Eqns (3.2) and (3.3), we obtain

$$
\begin{gathered}
-(2 n+1) \tilde{f}\{(Y \lambda) \eta(X)-(X \lambda) \eta(Y)\}=2 n(\lambda+1)\{\eta(X)(Y \tilde{f}) \\
-\eta(Y)(X \tilde{f})\}+(X \nu) \eta(Y)-(Y \nu) \eta(X) .
\end{gathered}
$$

Replacing $X$ by $\varphi X$ and $Y$ by $\xi$ in above relation, we get

$$
(2 n+1) \tilde{f} \varphi D \lambda+2 n(\lambda+1) \varphi D \tilde{f}-\varphi D \nu=0 .
$$

According to Proposition 2.1 of Chen [12], it is know that if $(\tilde{\xi} \tilde{f})=0$, then $\tilde{f}$ is constant. So that, Eqn (3.9) implies

$$
(2 n+1) \tilde{f} \varphi D \lambda=\varphi D \nu .
$$

The scalar curvature $r$ of $g$ is constant (as $(g, \lambda)$ is a solution of the CPE). For a $(2 n+1)$ dimensional $f$-cosymplectic manifold, we have $\nu=-r\left(\frac{\lambda}{2 n}+\frac{1}{2 n+1}\right)$, therefore from Eqn (3.10) it appears that

$$
\left((2 n+1) \tilde{f}+\frac{r}{2 n}\right) \varphi D \lambda=0 .
$$

From Eqn (3.11), we have either $r=-2 n(2 n+1) \tilde{f}$ or $\varphi D \lambda=0$.

First suppose that $r=-2 n(2 n+1) \tilde{f}$, then we have $D \nu=-(2 n+1) \tilde{f} D \lambda$. Plugging $X=\xi$ in Eqn (3.8) and calling back Lemma 3.1, we aimed at obtaining

$$
R(X, \xi) D \lambda=-2 n \tilde{f}(X \lambda)-(\xi \lambda) Q X+(\lambda+1)\{f Q X+2 n \tilde{f} f X\}+(X \nu)-(\xi \nu) X .
$$

From Eqn (3.3), we deduce $R(X, \xi) Y=\tilde{f}\{g(X, Y) \xi-\eta(Y) X\}$, by virtue of this the foregoing equation reduces to

$$
\begin{gathered}
-(2 n+1) \tilde{f}(X \lambda) \xi+(\tilde{f}(\xi \lambda)-(\xi \nu)+2 n \tilde{f} f(\lambda+1)) X \\
+(f(\lambda+1)-(\xi \lambda)) Q X+(X \nu) \xi=0 .
\end{gathered}
$$

Making use of $D \nu=-(2 n+1) \tilde{f} D \lambda$ in Eqn (3.12) we reach at

$$
(f(\lambda+1)-(\xi \lambda))(Q X+2 n \tilde{f} X)=0 .
$$

Since $\nabla_{\xi} \xi=0$ and $(\xi \lambda)=g(\xi, D \lambda)$, taking into account $\nabla_{X} D \lambda=(\lambda+1) Q X+\nu X$, we deduce $\xi(\xi \lambda)=f \lambda$. If possible, let $(\xi \lambda)=f(\lambda+1)$ in some open set $\mathcal{O}$ of $M$, then we have $f \lambda=\left((\xi f)+f^{2}\right)(\lambda+1)$. By virtue of $\tilde{f}=(\xi f)+f^{2}$, one can see $\lambda=\lambda+1$, that is, $1=0$, which is absurd. Hence $Q X=-2 n \tilde{f} X$ and $M$ is Einstein.

Next we assume $r \neq-2 n(2 n+1) \tilde{f}$, then from Eqn (3.11) we have $\varphi D \lambda=0$. Action of $\varphi$ on this equation gives $D \lambda=(\xi \lambda) \xi$. Differentiating this along $X$, calling back Eqn (3.1) furnishes

$$
\nabla_{X} D \lambda=X(\xi \lambda) \xi-f(\xi \lambda) \varphi^{2} X
$$

On the other hand, from Eqn (1.2) we can easily find that

$$
\nabla_{X} D \lambda=(\lambda+1) Q X+\left(\Delta \lambda-\frac{r}{2 n+1}\right) X
$$


Comparing aforementioned equation with Eqn (3.13), we get

$$
(\lambda+1) Q X+\left(\Delta \lambda-\frac{r}{2 n+1}\right) X=X(\xi \lambda) \xi-f(\xi \lambda) \varphi^{2} X .
$$

Taking $X=\xi$ in the above equation and making use of Eqns (3.2) and (2.1), we obtain

$$
\xi(\xi \lambda)=\left(\Delta \lambda-\frac{r}{2 n+1}\right)-2 n \tilde{f}(\lambda+1) .
$$

Contraction of Eqn (3.13) with respect to $X$ brings into view

$$
\Delta \lambda=\xi(\xi \lambda)+2 n f(\xi \lambda)
$$

Unifying this with Eqn (3.15) implies

$$
2 n f(\xi \lambda)-\frac{r}{2 n+1}-2 n \tilde{f}(\lambda+1)=0 .
$$

Differentiating Eqn (3.17) along $\xi$, keeping in mind that $\tilde{f}$ and $r$ are constants, we obtain $\xi(\xi \lambda) f+(\xi \lambda)(\xi f)=\tilde{f}(\xi \lambda)$, and further, it implies

$$
\xi(\xi \lambda)=f^{2}(\xi \lambda)
$$

where we used $\tilde{f}=(\xi f)+f^{2}$.

If $f \not \equiv 0$, then we can assume $f \neq 0$ on some neighborhood $\mathcal{O}$ of $M$. Thus, Eqn (3.18) implies $\xi(\xi \lambda)=(\xi \lambda) f$ on $\mathcal{O}$. Inserting this into Eqn (3.16), we find $\Delta \lambda=(2 n+1) f(\xi \lambda)$. Moreover, applying Eqn (3.17) in the previous relation shows that

$$
\Delta \lambda=(2 n+1)\left\{\tilde{f}(\lambda+1)+\frac{r}{2 n+1}\right\}
$$

Taking trace of $\mathrm{CPE}$ (1.2), we obatin $2 n \Delta \lambda=-\lambda r$, and this together with Eqn (3.19) gives that $r=-2 n(2 n+1) \tilde{f}$, which is contradictory to our assumption. Hence $f \not \equiv 0$, and so $M$ is cosymplectic. According to Blair's [13] result, we can easily conclude that $M$ is locally the product of a Kähler manifold and an interval or unit circle $\mathbb{S}^{1}$. This finishes the proof.

In particular, when dimension of $M$ is three, due to Theorem 3.3 we have the following outcome:

Corollary 3.4. Let $M$ be an $f$-cosymplectic manifold of dimension three satisfying $\mathrm{CPE}$ Eqn (1.2). If $(\tilde{\xi})=0$, then $M$ is either locally the product of a Kähler manifold and an interval or unit circle $\mathbb{S}^{1}$ or $M$ has constant negative sectional curvature $-\tilde{f}$.

It is known that an $\alpha$-cosymplectic manifold is actually an $f$-cosymplectic manifold with $f$ constant. By the reason of this, we obtain the following conclusion from Theorem 3.3.

Corollary 3.5. Let $M$ be an $\alpha$-cosymplectic manifold of dimension $2 n+1$ with $\xi(\tilde{f})=0$. If $(g, \lambda)$ is a solution of the CPE Eqn (1.2), then $M$ is either Einstein or locally the product of a Kähler manifold and an interval or unit circle $\mathbb{S}^{1}$.

Now we consider CPE with $\lambda=\tilde{f}$, and obtain the following result.

Theorem 3.6. If a connected $f$-cosymplectic manifold $M$ satisfying CPE Eqn (1.2) with $\lambda=\tilde{f}$, then $M$ is Einstein. 
Proof. One can easily obtain from Eqn (3.9) that

$$
\{(4 n+1) \lambda+2 n\} \varphi D \lambda=\varphi D \nu
$$

where we applied our assumption $\lambda=\tilde{f}$. Uptaking $\nu=-r\left\{\frac{\lambda}{2 n}+\frac{1}{2 n+1}\right\}$ in the above relation implies

$$
\left\{(4 n+1) \lambda+2 n+\frac{r}{2 n}\right\} \varphi D \lambda=0
$$

Suppose that $(4 n+1) \lambda+2 n+\frac{r}{2 n} \not \equiv 0$. Due to constancy of $r$, we see that $\lambda$ is constant. Next, we assume that $(4 n+1) \lambda+2 n+\frac{r}{2 n} \not \equiv 0 \$$ in a neighborhood $\mathcal{O}$ of $M$. Consequently, one can gets $\varphi D \lambda=0$. Applying $\varphi$ to this equation implies $D \lambda=(\xi \lambda) \xi$. In this context (3.13) holds, from which we can get

$$
2 n f(\xi \lambda)-\frac{r}{2 n+1}-2 n \lambda(\lambda+1)=0 .
$$

Differentiating this along $\xi$ gives $(2 \lambda+1)(\xi \lambda)=\xi(\xi \lambda) f+(\xi \lambda)(\xi f)$, due to our assumption $\lambda=\tilde{f}=(\xi f)+f^{2}$ which further implies

$$
\left(\lambda+f^{2}+1\right)(\xi \lambda)=\xi(\xi \lambda) f .
$$

Suppose that $f=0$, then from $\operatorname{Eqn}(3.20)$, we have $\lambda(\lambda+1)+\frac{r}{2 n(2 n+1)}=0$, which means that $\lambda$ is constant. In the following we suppose $f \neq 0$, then as a result of Eqns (3.16), (3.20) and (3.21), we find

$$
\Delta \lambda=\left\{(\lambda+1)+f^{2}(2 n+1)\right\} \frac{(\xi \lambda)}{f} .
$$

Substitute this into $2 n \Delta \lambda=-\lambda r$ to obtain

$$
-2 n \frac{(\xi \lambda)}{f}-2 n(2 n+1) \lambda=r .
$$

Differentiating the aforesaid relation along $\xi$, remembering $r$ is constant and applying Eqn (3.21), we reach at

$$
\left(2 n+3+\frac{1}{f^{2}}\right)(\xi \lambda)=0 .
$$

If $(\xi \lambda)=0$, then we have $D \lambda=0$, which means $\lambda$ is constant. Suppose $(\xi \lambda) \neq 0$, then we get $f^{2}=\frac{1}{2 n+3}$. Due to $f \neq 0$, which shows $(\xi f)=0$. This together with $\lambda=\tilde{f}=(\xi f)+f^{2}$ yields $\lambda=f^{2}$, showing $\lambda$ constant. In a word, we have proved that $\lambda$ is always constant in the neighborhood $\mathcal{O}$ of $M$, thus $\lambda=$ constant in $M$. Hence, the proof completes from Eqn (1.2).

\section{CPE on non-normal almost $f$-cosymplectic manifolds}

Here, we consider a three dimensional almost $f$-cosymplectic manifold $M$ with pseudo anticommuting Ricci tensor, that is,

$$
\varphi Q+Q \varphi=2 \kappa \varphi, \quad \kappa \text { is constant. }
$$

This notion was introduced by Jeong and Suh [14], and they made use of this condition to classify a real hypersurface of complex two-plane Grassmannians.

At first, we have the following lemma:
CPE on almost $f$-cosymplectic manifolds 
Lemma 4.1. [15] For a three dimensional almost $f$-cosymplectic manifold with pseudo anticommuting Ricci tensor the following formula holds:

$$
r-2 \kappa=a,
$$

where $a=g(Q \xi, \xi)$.

Let $\mathcal{U}$ be the open subset where the tensor $h \neq 0$ and $\mathcal{U}^{\prime}$ be the open subset such that $h$ is identically zero. Thus, $\mathcal{U} \cup \mathcal{U}$ is open dense in $M$. There exists a local orthonormal frame field $E=\{\xi, e, \varphi e\}$ such that $h e=\mu e$ and $h \varphi e=-\mu \varphi e$, where $\mu$ is a positive nonvanishing smooth function of $M$. The following proposition is obtain from Proposition 12 and Proposition 14 of Öztürk et al. [10]:

Proposition 4.2. For a three dimensional almost $f$-cosymplectic manifold, the following relations hold:

$$
\begin{gathered}
h^{2}-f^{2} \varphi^{2}=\frac{a}{2} \varphi^{2}, \\
\nabla_{\xi} h=2 b h \varphi+(\xi \mu) s,
\end{gathered}
$$

where $b$ is a function defined by $b=g\left(\nabla_{\xi} \varphi e, e\right)$ and $s$ is a $(1,1)$ tensor field defined by $s e=e$, $s \varphi e=-\varphi e$ and $s \xi=0$.

From this onwards, we assume that a three dimensional almost $f$-cosymplectic manifold $M$ satisfies the CPE Eqn (1.2), then its scalar curvature $r$ is constant. As a result of Lemma 4.1, it can be seen that $a$ is constant. Chen in [15] obtained the relation $(r-2 \kappa-2 \tilde{f}-2 a) \varphi X=2 \varphi h^{2} X$. From this we have $(-2 \tilde{f}-a) \varphi X=2 \varphi h^{2} X$, that is, $2 h^{2} X=(2 \tilde{f}+a) \varphi^{2} X$ as $h \xi=0$. Further, from Eqn (4.1) we find $(\xi f)=0$, due to $d f \wedge \eta=0$ we obtain $f$ is constant. Moreover, we can also find $a=-2\left(f^{2}+\mu^{2}\right)$ from Eqn (4.1). Thus $\mu$ is constant. In view of Lemma 2 of [10], we have the following (also see [15]):

Lemma 4.3. Let $M$ be a three dimensional almost $f$-almost cosymplectic manifold satisfying the CPE Eqn (1.2). If the Ricci tensor is pseudo anticommuting, then with respect to $E$ the Levi-Civita connection $\nabla$ is given by

$$
\begin{array}{cl}
\nabla_{\xi} e=-b \varphi e, & \nabla_{\xi} \varphi e=b e, \quad \nabla_{\xi} \xi=0, \\
\nabla_{e} \xi=f e-\mu \varphi e, & \nabla_{e} e=-f \xi, \quad \nabla_{e} \varphi e=\mu \xi, \\
\nabla_{\varphi e} \xi=-\mu e+f \varphi e, & \nabla_{\varphi e} \varphi e=-f \xi, \quad \nabla_{\varphi e} e=\mu \xi .
\end{array}
$$

In view of Eqn (4.2), the relation Eqn (2.5) implies

$$
R(X, \xi) \xi=-\frac{a}{2} \varphi^{2}+2 f \varphi h X+2 b h X .
$$

By virtue of Eqn (3.8), we obtain

$$
\begin{aligned}
g(R(X, \xi) D \lambda, \xi) & =a(X \lambda)-a(\xi \lambda) \eta(X)+(X \nu)-(\xi \nu) \eta(X) \\
& =\left(a-\frac{r}{2}\right)((X \lambda)-(\xi \lambda) \eta(X)) .
\end{aligned}
$$


Substituting the above relation into Eqn (4.4), we obtain

$$
\begin{gathered}
\frac{a}{2} g\left(\varphi^{2} X, D \lambda\right)-2 f g(\varphi h X, D \lambda)-2 b g(h X, D \lambda) \\
=\left(a-\frac{r}{2}\right)((X \lambda)-(\xi \lambda) \eta(X)) .
\end{gathered}
$$

Employing $X$ by $\varphi X$ in Eqn (4.6) we reach at

$$
\left(\frac{3 a-r}{2}\right) \varphi D \lambda=2 f h D \lambda+2 b h \varphi D \lambda
$$

In orthonormal frame field $E$, the gradient vector field $D \lambda$ can be written as

$$
D \lambda=(e \lambda) e+(\varphi e \lambda) \varphi e+(\xi \lambda) \xi .
$$

Thus from Eqn (4.7), one can obtain

$$
\begin{gathered}
-\left(\frac{3 a-r}{2}\right)(\varphi e \lambda)=2 f \mu(e \lambda)-2 b h(\varphi e \lambda), \\
\text { and }\left(\frac{3 a-r}{2}\right)(e \lambda)=-2 f \mu(\varphi e \lambda)+2 b h(e \lambda) .
\end{gathered}
$$

First we assume $f \neq 0$, because of $f$ is constant and we shall divide this discussion into two cases:

Case 1. If $(e \lambda)=0$, then from Eqn (4.10) we can observe $(\varphi e \lambda)=0$. This together with (4.8) yields $D \lambda=(\xi \lambda) \xi$. Differentiating this along $X$, using Eqn (2.2) gives

$$
\nabla_{X} D \lambda=X(\xi \lambda)-(\xi \lambda)\left(f \varphi^{2} X+\varphi h X\right) .
$$

Employing $X=\xi$ in the above equation and remembering $\nabla_{\xi} D \lambda=(\lambda+1) Q \xi+\left(\Delta \lambda-\frac{r}{3}\right) \xi$, we aimed at obtaining

$$
\xi(\xi \lambda)=(\lambda+1) a+\Delta \lambda-\frac{r}{3}
$$

One can find from Eqn (4.11) and second term of Eqn (2.2) that

$$
\Delta \lambda=\xi(\xi \lambda)+2 f(\xi \lambda) .
$$

By virtue of the foregoing relation, Eqn (4.12) transforms into $(\xi \lambda)=\frac{r}{6 f}-\frac{(\lambda+1) a}{2 f}$, which further gives

$$
\xi(\xi \lambda)=-\frac{(\xi \lambda) a}{2 f}=-\frac{r a}{12 f^{2}}+\frac{(\lambda+1) a^{2}}{4 f^{2}}
$$

where we applied $f$ and $a$ are constants. Also, it is know that $\Delta \lambda=-\frac{\lambda r}{2}$. Thus Eqn (4.12) transforms into

$$
\lambda\left(\frac{a^{2}}{4 f^{2}}+\frac{r}{2}-a\right)=\frac{r a}{12 f^{2}}-\frac{r}{3}-\frac{a^{2}}{4 f^{2}}+a
$$

CPE on almost $f$-cosymplectic manifolds 
If $\frac{a^{2}}{4 f^{2}}+\frac{r}{2}-a=0$, then we have $\frac{r a}{12 f^{2}}-\frac{r}{3}-\frac{a^{2}}{4 f^{2}}+a=0$, which further shows that $\frac{r}{6}\left(\frac{a}{2 f^{2}}+1\right)=0$. The former case implies that the scalar curvature of $M$ vanishes identically. In the latter case, we have $a=-2 f^{2}$, which together with $a=-2\left(f^{2}+\mu^{2}\right)$ implies $\mu=0$, which is not possible. Next suppose $\frac{a^{2}}{4 f^{2}}+\frac{r}{2}-a \neq 0$, then from Eqn (4.14) it can easily conclude that $\lambda$ is constant.

Case 2. If $(e \lambda) \neq 0$ on a neighborhood $\mathcal{O}$ of $M$, then from (4.9) and (4.10) we extract

$$
\left(\frac{3 a-r}{2}\right)^{2}=4\left(f^{2}+b^{2}\right) \mu^{2}
$$

From the preceding equation, we can easily observe that $b$ is constant because of $\mu$ and $f$ are constant. It is easy to seen from Eqns (4.9) and (4.10) that $(e \lambda)$ and $(\varphi e \lambda)$ are constants in $\mathcal{O}$.

By the support of Eqn (4.3), we may easily compute that

$$
\begin{aligned}
& \nabla_{e} D \lambda=(-f(e \lambda)+\mu(\varphi e \lambda)+e(\xi \lambda)) \xi+(\xi \lambda)(f e-\mu \varphi e), \\
& \nabla_{\varphi e} D \lambda=(\mu(e \lambda)-f(\varphi e \lambda)+\varphi e(\xi \lambda)) \xi+(\xi \lambda)(-\mu e+f \varphi e), \\
& \nabla_{\xi} D \lambda=-b(e \lambda) \varphi e+b(\varphi e \lambda) e+\xi(\xi \lambda) \xi .
\end{aligned}
$$

Thus $\Delta \lambda=2 f(\xi \lambda)+\xi(\xi \lambda)$. So, utilization of $\nabla_{\xi} D \lambda=(\lambda+1) Q \xi+\left(\Delta \lambda-\frac{r}{3}\right) \xi$ followed from (3.14), shows that

$$
(\xi \lambda)=\frac{r}{6 f}-\frac{(\lambda+1) a}{2 f} .
$$

As followed by Case 1, we can conclude that either $r$ vanishes or $\lambda$ is constant.

Next we assume $f=0$, then from Eqns (4.9) and (4.10) we find $b(e \lambda)(\varphi e \lambda)=0$ as $\mu>0$, which further implies either $(e \lambda)(\varphi e \lambda)=0$ or $b=0$. We shall also discuss this matter into two cases.

Subcase $i$. If $b=0$, then from Eqn (4.10) we find $r=3 a$. For three dimensional case, it is known that the Riemannian curvature is

$$
\begin{aligned}
R(X, Y) Z= & g(Y, Z) Q X-g(X, Z) Q Y+g(Q Y, Z) X-g(Q X, Z) Y \\
& -\frac{r}{2}\{g(Y, Z) X-g(X, Z) Y\} .
\end{aligned}
$$

From this, we have

$$
\begin{aligned}
R(X, \xi) \xi & =Q X-2 a \eta(X) \xi+a X-\frac{r}{2}\{X-\eta(X) \xi\} \\
& =Q X-\frac{a}{2} \eta(X) \xi-\frac{a}{2} X .
\end{aligned}
$$

This together with Eqn (4.4) yields $Q X=a X$, which means $M$ is Einstein.

Subcase $i i$. If $b \neq 0$ on some neighborhood $\mathcal{O}$ of $M$, then we find $(e \lambda)(\varphi e \lambda)=0$ on $\mathcal{O}$. If possible, let $(e \lambda)=0=(\varphi e \lambda)$, then from Eqn (4.8) we obtain $D \lambda=(\xi \lambda) \xi$. From this it is not hard to see that Eqn (4.12) holds and Eqn (4.13) implies $\Delta \lambda=\xi(\xi \lambda)$. Thus Eqn (4.12) transforms into $(\lambda+1) a-\frac{r}{3}=0$, which means $\lambda$ is constant on $\mathcal{O}$.

If $(e \lambda)=0$ and $(\varphi e \lambda) \neq 0$, then from Eqns (4.3) and (4.8) we compute

$$
\nabla_{\xi} D \lambda=\xi(\varphi e \lambda) \varphi e+b(\varphi e \lambda) e+\xi(\xi \lambda) \xi .
$$


Utilization of above relation in $\nabla_{\xi} D \lambda=\left(\Delta \lambda-\frac{r}{3}\right) \xi+(\lambda+1) a \xi$, we find $b(\varphi e \lambda)=0$. Because of $b \neq 0$, we have $(\varphi e \lambda)=0$, which is a contradiction. In a similar manner, we also come to contradiction if we consider $(e \lambda) \neq 0$ and $(\varphi e \lambda)=0$.

From the above detailed discussion, we have concluded that $M$ is Einstein or its scalar curvature vanishes, and so we state following result:

Theorem 4.4. Let $M$ be a three dimensional almost $f$-almost cosymplectic manifold satisfying the CPE Eqn (1.2), if its Ricci tensor is pseudo anti-commuting, then $M$ is either Einstein or its scalar curvature vanishes identically.

\section{References}

1. Besse A. Einstein manifolds. New York, NY: Springer; 2008.

2. Barros A, Ribeiro E Jr. Critical point equation on four-dimensional compact manifolds. Math Nachr. 2014; 287(14-15): 1618-1623.

3. Hwang, S. Critical points of the total scalar curvature functionals on the space of metrics of constant scalar curvature. Manuscripta Math. 2000; 103: 135-142.

4. Neto BL. A note on critical point metrics of the total scalar curvature functionals. J Math Anal Appl. 2015; 424: 1544-1548.

5. Ghosh A, Patra DS. The critical point equation and contact geometry. J Geom. 2017; 108: 185-194.

6. Patra DS et al The critical point equation on Kenmotsu and almost Kenmotsu manifolds. Publ. Math. Debrecen. 2020; 97(1-2): 85-99.

7. De UC, Mandal K. Critical point equation on almost Kenmotsu manifolds. Ukrainian Math. J. 2020; 72(1). doi: 10.1007/s11253-020-01770-5.

8. Naik, DM et al. Certaine types of metrics on almost coK’ahler manifolds. Ann. Math. Québec. 2020. (2021). doi: 10.1007/s40316-021-00162-w.

9. Blaga AM, Dey C. The Critical point equation on 3-dimensional $\alpha$-cosymplectic manifolds. Kyungpook Math J. 2020; 60: 177-183.

10. Öztürk H et al. Almost $\alpha$-cosymplectic $(\kappa, \mu, \nu)$-spaces. 2010. Available From: arXiv:1007.0527v1.

11. Aktan, $\mathrm{N}$ et al. Almost f-cosymplectic manifolds. Mediterr J Math. 2014; 11: 775-787.

12. Chen X. Notes on Ricci solitons in $f$-cosymplectic manifolds. J Math Phys Anal Geom. 2017; 13: 242-253.

13. Blair DE. Riemannian geometry of contact and symplectic manifolds. Progress Math. 2002; 203: Birkhäuser Boston.

14. Jeong I, Suh YJ. Pseudo anti-commuting and Ricci soliton real hypersurfaces in complex two-plane Grassmannians, J Geom Phys. 2014; 86: 258-272.

15. Chen X. On almost $f$-cosymplectic manifolds satisfying the Miao-Tam equation. J Geom. 2020; 111: 28. doi: 10.1007/s00022-020-00542-7.

\section{Corresponding author}

V. Venkatesha can be contacted at: vensmath@gmail.com

For instructions on how to order reprints of this article, please visit our website:

www.emeraldgrouppublishing.com/licensing/reprints.htm

Or contact us for further details: permissions@emeraldinsight.com 